\title{
Numerical Simulation of Internal Flow in "S" Shaped Outlet Conduit
}

\author{
Rongsheng Xie, Jiren Zhou, Fangping Tang *, Chao Liu, Lijian Shi \\ School of Hydraulic, Energy and Power Engineering, Yangzhou University, Yangzhou 225009, China \\ *Corresponding Author: tangfp@yzu.edu.cn
}

\begin{abstract}
Base on the stander $\mathrm{k}-\varepsilon$ turbulent model and Reynolds Navier-Stokes equation, the hydraulic performance of the $\mathrm{S}$-shaped shaft pumping station is simulated with the commercial CFD software, and the experiment results are compared with the CFD results. By comparing the relationship of the average swirl angel in the inlet and the hydraulic loss coefficient of the outlet conduit, the hydraulic performance of s-shaped outlet conduct is analyzed, we find that there is a optimal average swirl angel value for hydraulic loss coefficient. In this paper, the optimal average swirl angel is 5.3 degree, and the hydraulic loss coefficient is 0.000162 . By analyzing the static pressure and total pressure distribution curve, the conclusion below can be drawn: the reason for the large hydraulic loss is average swirl angel value is large at the small discharge, on the contrary the reason for the large hydraulic loss is velocity at large discharge.
\end{abstract}

Keywords: outlet conduit, hydraulic loss, swirl angle, CFD

\section{Introduction}

To ensure the efficient and stable, the tubular pump is used to minimize the hydraulic loss in large low-head pumping station. The energy is stored as pressure energy and kinetic energy of rotation when the water flow through the impeller of axial flow pump at high speed. Most of the kinetic energy can be recycled and translated into pressure energy with the effect of the guide vane. The small area of inlet, rotation water and the uneven distribution will result in the large velocity which will reduce gradually in the passage outlet on the inlet section of the passage outlet. In the passage outlet, part of the kinetic energy is converted into pressure energy, and the other part is lost because of the friction between the water and the fixed wall. The screw flow will happen when the water passes the spread channel with the influences of the velocity and circulation, the hydraulic loss is not simple direct ratio and velocity of secondary, but there is a optimal value.

The inlet and out conduits were designed separately in the early times, with the development of pump station, CFD is used to simulate the three-dimensional internal flow of low-lift pumping system including blades and guide vanes. Tang fangping quantitatively analyzed the recyclable energy with axial flow guide vane of different specific speed $^{[1]}$, so that the basis of circulation distribution on the inlet of passage outlet is drawn. In the outlet of large axial pump, there is optimal swirling flow which will make hydraulic losses of the singular tube discharge passage lowest and the method to solve the models and design the outlet diffuser is given ${ }^{[2]}$. Lu Weigang discussed the cross influence of discharge and circulation effecting the head loss of the conduit of pump system at low head ${ }^{[3]}$. By simulating and developing the passage, considering the Influence of flow velocity circulation at guide vane outlet of axial-flow pump on hydraulic loss in outlet conduit ${ }^{[4]}$, the hydraulic performance of low head pump station had a great improvement, the efficiency of shaft tubular pump system increased to $80 \%$, which can reach to $83 \%{ }^{[5]}$, and the s-shaped tubular pump system is $83.55 \%^{[6]}$.

In this paper, CFD method is used to simulate the internal flow in s-shaped outlet conduit, the hydraulic performance is analyzed.

\section{Mathematical model and Boundary conditions}

\subsection{Calculation model and parameters}

The diameter of the 3-bladed impeller is $300 \mathrm{~mm}$. The tip clearance of impeller is $0.2 \mathrm{~mm}$, rotational speed is 1022 $\mathrm{rpm}$. The calculation zone contains the inlet, outlet conduit, its extension, blade and guide vane. The length of the calculation model is $8 \mathrm{~m}$, the model and grid of the blade and guide vane are finished by the turbogrid software, the models of the conduit are made up by UG, and their structured grid were finished by ICEM. The quality of the grid is high than 0.35 , and the min angel is high than 24 . the number of separate part is shown in table 1 . The model and grid are shown in Fig.1. 


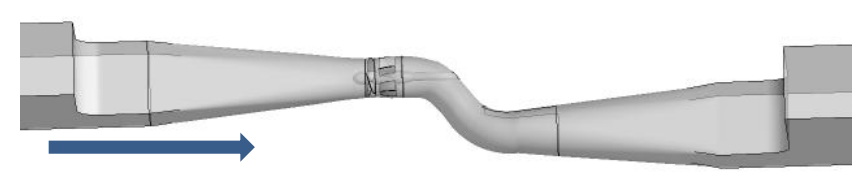

(a) Computational domain

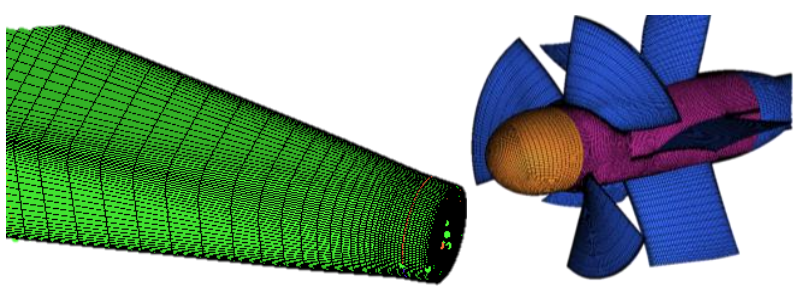

(b) inlet conduit grid

(c) blade and guide vane grid

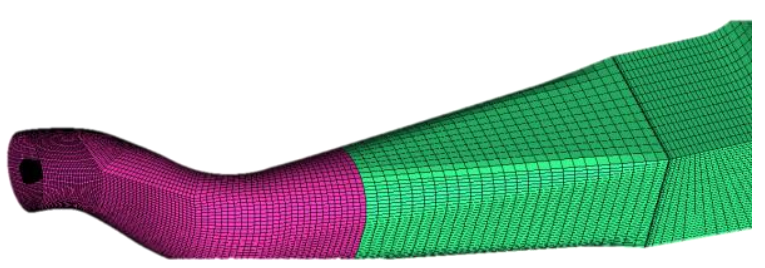

(d) outlet conduit grid

Fig.1. Computational domain and surface grid

Table 1 the nodes and elements

\begin{tabular}{|c|c|c|}
\hline Domain & Nodes & Elements \\
\hline Blade & 315612 & 292587 \\
\hline Guide vane & 291365 & 267210 \\
\hline Outlet conduit & 498420 & 478898 \\
\hline Intlet conduit & 200080 & 191089 \\
\hline All Domains & 1305477 & 1229784 \\
\hline
\end{tabular}

\subsection{Boundary conditions}

Base on the standard k- $\varepsilon$ turbulent model and Reynolds Navier-stokes equation ${ }^{[7]}$, the internal flow was calculated. The inlet boundary is set as total pressure, and mass-averaged flow rate is assumed on the outlet boundary. The stage interfaces were employed for calculations. The adiabatic and absolute no-slip boundary conditions are applied for the solid walls of the runner chamber, while relative no-slip boundary conditions for the other solid walls ${ }^{[8]}$. The toque of the blade and the delta total pressure between the inlet and outlet domain were monitor when the calculator is running. When each of the monitors reached a stable number, the simulation is completed.

\section{Results and discussion}

\subsection{Comparisons between predictions and test results}

The model test was taken on Hydrodynamic Engineering Laboratory of Jiangsu Province, Yangzhou University, according to water conservancy industry standard of PRC 《Code for model pump and its installation acceptance tests》(SL140-2006). Fig 2 is the picture of model pump station.

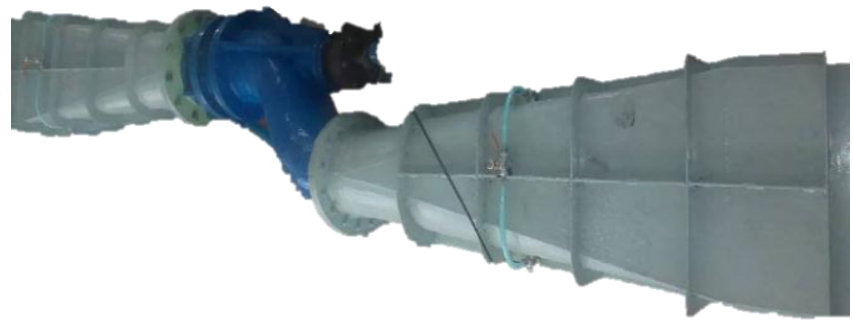

Fig.2. The picture of model pump systems

In order to ensure accuracy of calculation, performance curve of the Power distribution vs flow $Q$ and Head distribution vs flow $Q$ under different blade anger are shown Fig. 3, Fig. 4.

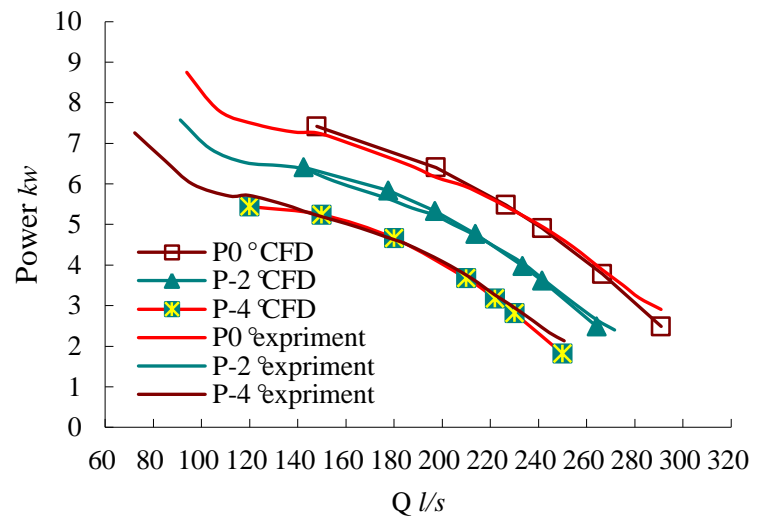

Fig.3. Power distribution vs flow rate $Q$

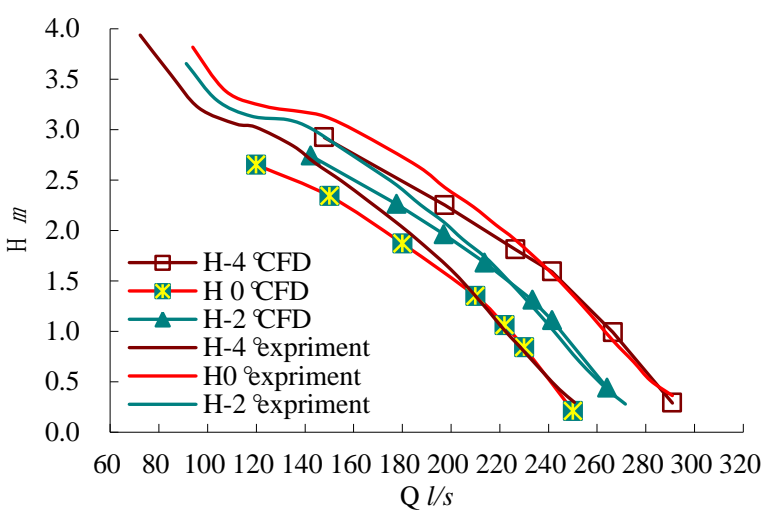

Fig.4. Head distribution vs flow rate $Q$ 
As Fig.3 shown, it can be seen that the relationship between power and flow is almost coincided with test result, the slope of the calculated curve had a little steep than test result. As Fig. 4 shown, it can be seen that the relationship between head and flow is coincided in large flow rate, the calculate head is lower than the test result, the overall curve trend consistent. Simulation results to be trusted.

\subsection{Analysis of hydraulic loss in outlet conduit}

To calculate the hydraulic loss between the inlet and outlet section of the s-shaped conduit, the relationship curve between hydraulic loss and flow is given which is shown in Fig 5.

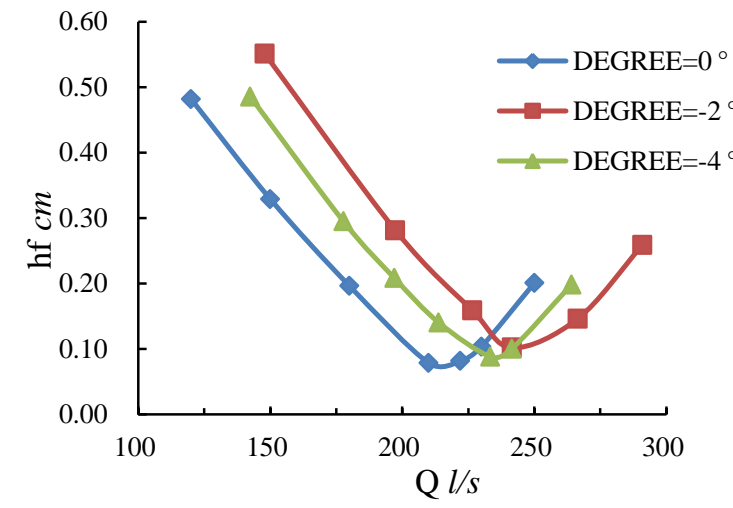

Fig.5. Hydraulic loss distribution vs flow $Q$

From Fig.5, it is shown that hydraulic loss in outlet conduit is not a simple relationship with flow into a monotone increasing, but has a certain minimum value. This minimum value of the flow with the decrease of the corresponding with the decrease of the blade angle. In order to find the relationship between the hydraulic loss and flow rate, the concept of average swirl angle is introduced, which can be defined as the arctangent value of Average tangential velocity and average axial velocity ${ }^{[9]}$, at the same time, the loss coefficient $\zeta$ is defined as the hydraulic loss of discharge passage divided by the square of flow. Those can be written as equation (1) and (2).

$$
\begin{aligned}
& \bar{\theta}=\operatorname{atan} \frac{\overline{v_{t}}}{\overline{v_{a}}} \\
& \zeta=\frac{\boldsymbol{h}_{f}}{\boldsymbol{Q}^{2}}
\end{aligned}
$$

To set the discharge as the abscissa, average swirl angle in the inlet section of the outlet conduit as the ordinate, we can draw Fig.6. To set average swirl angle as the abscissa, hydraulic loss coefficient $\zeta$ as the ordinate, we can draw Fig.7.

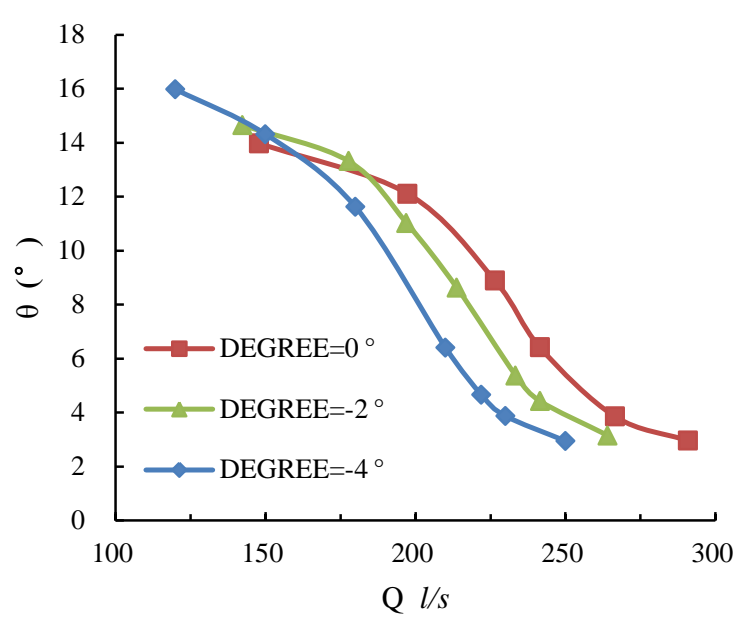

Fig.6. Average swirl angle $\bar{\theta}$ vs flow rate $Q$

As Fig 6 shown, the curves of the average flow and swirl angle have the same trend in different blade Angle. The average swirl Angle decreases as the discharge increases. As the discharge increases, the head reduces, the rest of the outlet circulation after the guide vane decreases, and the average swirl angle decrease.

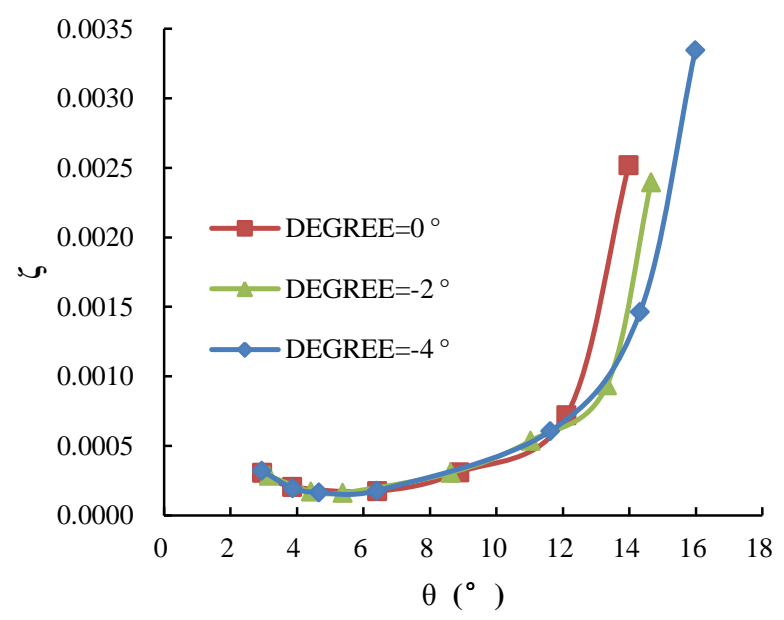

Fig.7. Average swirl angler $\bar{\theta}$ vs head loss

As Fig.7 shown, the hydraulic loss coefficient will reduce at first and then increase when the vertex angle increase. The increase of vertex angle will reduce the diversion loss before the vertex angle is less than the best vertex angle. The friction loss will increase when the swirl angle increase which the flow distance is lengthen. The major reason of the hydraulic loss coefficient increase rapidly is the serious separation flow when the vertex angle increases step by step. With the decrease of the blade Angle, the position of the inflection point appears biased vortex Angle larger area.

In order to further study the relationship between the hydraulic loss coefficient and swirl Angle, the first blade (blade1) to another blade (blade2) which has the bigger 
specific speed is changed. Fig. 8 gives relationship between the average swirl angle and the head loss under different blades.

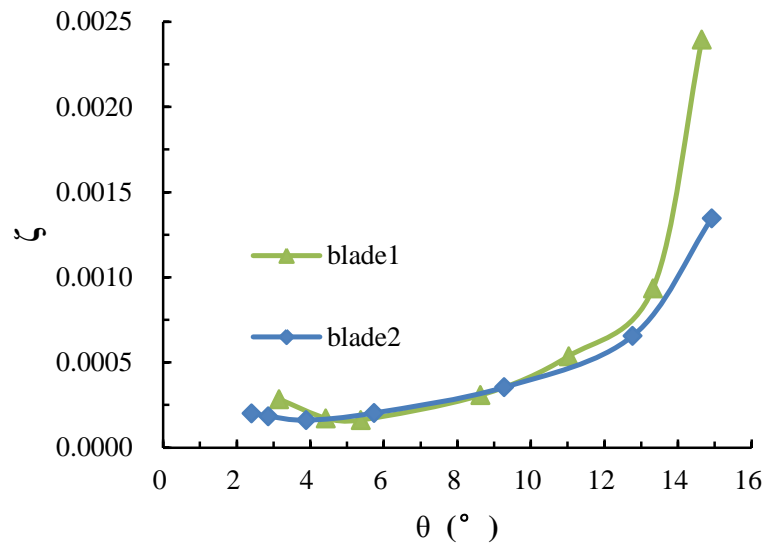

Fig.8. The relationship between the average swirl angle $\bar{\theta}$ and the head loss under different blade

As figure 8 shown, the average swirl angle and hydraulic loss coefficient in larger range after the impeller is replaced, the relationship between hydraulic loss coefficient and average swirl angle of S-shaped is mainly related to the structure of the passage itself.

By changing the blade, the optimal average swirl turn to a small angle.

\subsection{Analysis of pressure distribution}

To set the inlet section as the first plane, insert the vertical section with the center line of the passage, and draw the total pressure and static pressure along with the center line of the passage which shown as Fig.9.

Base on the surface of the outlet conduit, offset it within $2 \mathrm{~cm}, 4 \mathrm{~cm}, 8 \mathrm{~cm}$ in turn, we can get 4 surface from the outside to inside, paint the surface pressure distribution on the surface, shown as Fig 10.

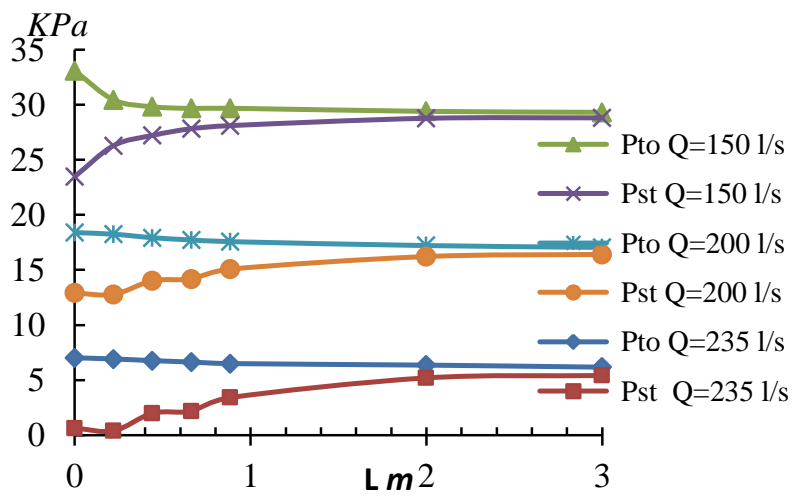

Fig. 9. The curves of $P_{t o}$ and $P_{s t}$ along with the center line

As figure 9 shown, along with the center line of the passage, the hydraulic loss gradually increase, the total pressure decreases gradually; the area of each section gradually expanded, conduit recycling kinetic energy into pressure, static pressure is gradually increased. In the s-shaped outlet conduit, the water is able to adjust, most of the kinetic energy is recycled, static pressure increases obviously, as a result the hydraulic loss is also bigger. By comparing the kinetic energy in the inlet section under difference flow rate, it is found that the kinetic energy is big under undersign condition, it will cause more loss when translation to static pressure. The major reason for the hydraulic loss is the circulation at low flow condition, and the major reason for the hydraulic loss is the flow at larger flow condition.

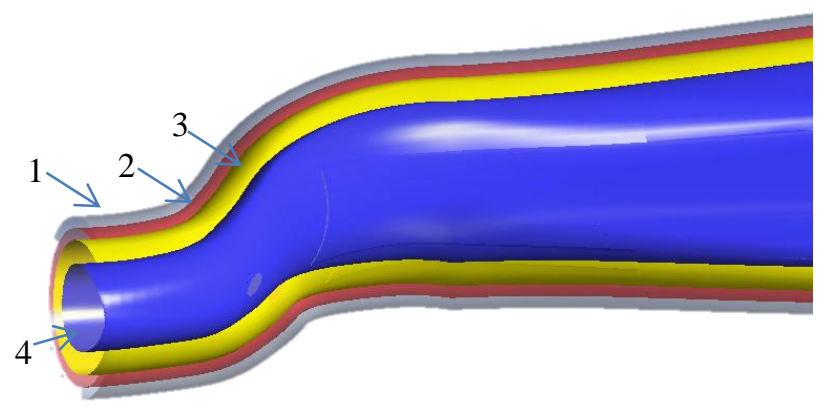

The figure of offset surface

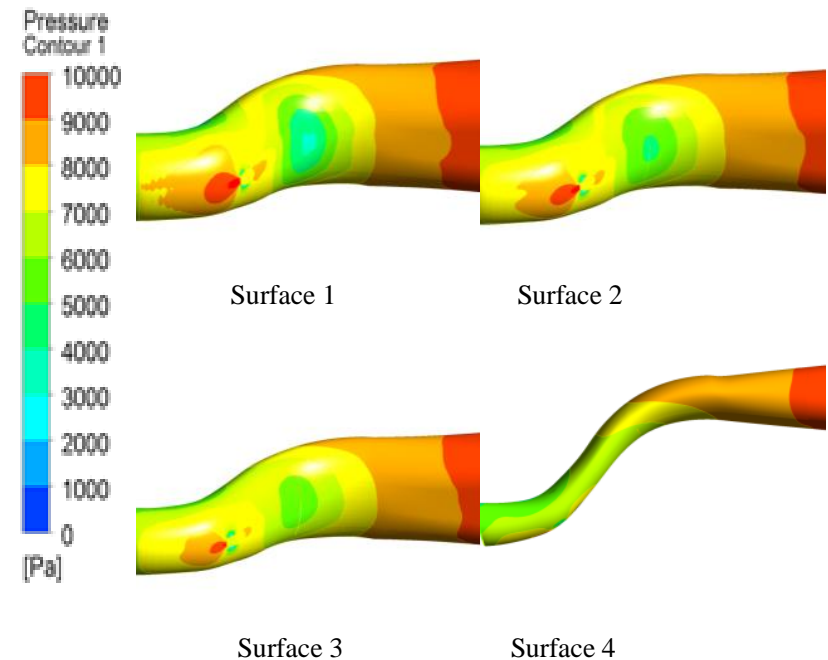

Fig.10. The distribution of pressure $\quad(\mathrm{Q}=222 \mathrm{l} / \mathrm{s})$

Overall pressure on the inlet section is low, as with the section area increasing, the pressure increasing. Due to the influence of the axial and the bending flow passage, there is a local pressure increase in the first corner out, and decrease after through the axial. Between the first corner and second, the pressure from high to low near to axial, from low to high far away the axial. The cross section flow to readjust under the influence of the s-shaped passage, most of the circulation is recycled. 


\section{Conclusions}

1. The hydraulic loss distribution is drawn by simulation of S-shaped shaft pumping station with different blade angels and different blades, the hydraulic loss coefficient $\zeta$ mainly related to average swirl angel $\bar{\theta}$ and the structure of the passage itself. Blade angel and blade have litter effect on the minimum hydraulic loss coefficient

2. The optimal swirl Angle in the inlet of the s-shaped conduit between $4^{\circ}$ and $5.5^{\circ}$, the minimum hydraulic loss coefficient $\zeta$ is about 0.000162 .

3. Most of the circulation is able to recycle after the water flowing though the two corners of the S-shaped outlet conduit.

4. S-shaped outlet conduit has a low hydraulic loss at the design condition because of the low kinetic energy on the inlet section which will cause less loss when translated to static pressure. The circulation is the major reason for the hydraulic loss at low discharge condition, and the flow is the major reason for hydraulic loss at larger discharge condition.

\section{References}

(1) Tang Fangping, Zhou Jiren, Yan Bipeng. Analysis of Recycled circulation by axial flow pump back guide vane[J].Pump technology, No.03.pp.19-22,1995.

(2) Qiu Baoyun, Liu Chao, Yuan Weisheng. Influence of Blade Outlet Angel of Axial Outlet Diffuser of Large Pump on Performance of Its Discharge Passage[J].Journal of Mechanical Engineering No.07 pp.74-77, 2000.

(3) LU Wei- gang, DONG Lei, WANG Zhaofei, LU Linguang. Cross influence of discharge and circulation on head loss of the conduit of pump system with low head [J], Applied Mathematics and Mechanics, Vol.33,No.12, pp.1431-1441, Dec.15,2012.

(4) Liang Jindong, Lu Linguang, Xu Lei, Chen Wei, Wang Gang. Influence of flow velocity circulation at guide vane outlet of axial-flow pump on hydraulic loss in outlet conduit [J], Transactions of the CSAE ,Vol.28, No.1, pp.55-60, 2012.

(5) Xu Lei, Lu Linguang, Chen Wei, et al. Flow pattern analysis on inlet and outlet conduit of shaft tubular pump system of Pizhou pumping station in South-to-North Water Diversion Project[J],Transactions of the CSAE, Vol.28, No.6, pp.50-56. 2012.

(6) Yang Fan, Liu Chao, Tang Fangping, et al. Hydraulic Performance Analysis of Flow Passage Components in S-shaped Shaft Extension Tubular Pumping System under Multi-conditions $[\mathbf{J}]$. Transactions of the Chinese Society for Agricultural Machinery, Vol.45, No.5, pp. 71-77, 2014.

(7) Blazek J. Computational Fluid Dynamics: Principles and Applications [M] . 2nd ed. Netherlands: Elsevier Ltd. pp 227-270. 2005.

(8) Rodi W. Turbulence Models and Their Application in Hydraulics Experimental and Mathematical Fluid Dynamics [M] .Delft: IAHR Section on Fundamentals of Division II. pp .44-46, 1980.

(9) ANSI/HI 9.8-1998. American National Standard for Pump Intake Design $[S]$. 